\title{
Psicooncología
}

ISSN: $1696-7240$

https://dx.doi.org/10.5209/psic. 77754

\section{Variables predictivas de calidad de vida en mujeres con cáncer de mama}

\author{
Ana Luz Protesoni ${ }^{1 *}$; Sofía Grille ${ }^{2}$
}

Recibido: 10 de julio de 2021 / Acepado: 20 de agosto de 2021

Resumen. Objetivos: Estudiamos el valor predictivo que tiene sobre la calidad de vida relacionada con la salud (CVRS) de las mujeres con cáncer de mama en tratamiento, las variables sociodemográficas, clínicas (tipo de tratamiento) y psicológicas tales como los esquemas desadaptativos tempranos (EDT), las creencias y percepciones sobre la enfermedad y las estrategias de afrontamiento utilizadas. Métodos: Realizamos un estudio descriptivo, correlacional, de subtipo predictivo. Incorporamos una muestra de 228 pacientes adultas con cáncer de mama en tratamiento ambulatorio del sistema público asistencial. Se realizaron entrevistas en las que se aplicó un formulario de datos socio demográficos y clínicos, el Cuestionario FACT-B, el Test de Esquemas Tempranos (YSQ-CEY-VR), el Cuestionario de percepción de enfermedad (IPQ-R) y la escala Brief COPE. Se realizaron análisis descriptivos, de estadística inferencial, regresión lineal múltiple y regresión jerárquica. Resultados: Los diferentes tipos de tratamientos onco-específicos no registraron diferencias significativas en la CVRS, sin embargo las mujeres con mastectomía sin reconstrucción valoraron sensiblemente descendida la CVRS con respecto a los otros tipos de cirugía. Las variables que mostraron relación estadística en explicar el FACT-B global fueron los esquemas de Deprivación emocional, Abandono, Imperfección/vergüenza, Vulnerabilidad, Apego, Dominio 3 y Dominio 4. Así también todos los valores del IPQ-R guardaron relación significativa con la CVRS global. Las creencias y percepción global de la enfermedad (IPQR) y los dominios de los EDT, tienen valor predictivo sobre CVRS. Conclusiones: las mujeres con una percepción amenazante de la enfermedad tienden a activar pautas de funcionamiento caracterizadas por la desconexión emocional, el aislamiento, la vergüenza; u otras caracterizadas por la dependencia, la pérdida de autonomía y confianza en sí mismas. Las creencias y percepciones sobre la enfermedad activan necesidades y pautas de respuestas cognitivas, emocionales y relacionales (esquemas desadaptativos) siendo ambas variables predictivas del mayor o menor ajuste y percepción global de bienestar.

Palabras clave: Cáncer de mama, calidad de vida, esquemas desadaptativos tempranos, creencias

\section{[en] Predictive variables of quality of life in women with breast cancer}

Abstract. Objective: We studied the predictive value of sociodemographic, clinical (type of treatment) and psychological variables such as early maladaptive schemas, beliefs and perceptions about the disease and the coping strategies used by patients on health-related quality of life (HRQOL) of women with breast cancer under treatment. Method: We conducted a descriptive and correlational predictive study. Two hundred twenty-eight adult breast cancer patients under outpatient treatment from the

1 Ana Luz Protesoni. Facultad de Psicología. Universidad de la República. Montevideo. Uruguay.

E-mail: protesoni@psico.edu.uy

2 Sofía Grille. Facultad de Medicina. Universidad de la República. Montevideo. Uruguay.

E-mail:sofiagrille@gmail.com

* Dirección de correspondencia: Ana Luz Protesoni. Tristán Narvaja 1674 CP 11200 - Montevideo - Uruguay. Email: protesoni@psico.edu.uy 
public healthcare system were included. Interviews were conducted and sociodemographic and clinical data were collected. Several questionnaires were applied: FACT-B questionnaire, early maladaptive schemas form, Brief Illness Perception Questionnaire (IPQ-R) and COPE dispositional questionnaires. Results: Descriptive and inferential analysis, multiple linear regression, and hierarchical regression were performed. Oncology treatments subtypes did not influence HRQoL. However, women who had undergone a mastectomy without reconstruction showed significantly decreased HRQoL values. Variables that showed a statistical relationship in explaining the global FACT-B were: schemes of emotional deprivation, abandonment, defectiveness/shame, vulnerability, attachment, domain 3 and domain 4. Also, all the IPQ-R values were significantly related to the Global HRQoL. The beliefs and global perception of the disease (IPQ-R) and EDT domains, have shown predictive value on HRQL. In conclusion, women with a threatening perception of the disease tended to activate functional patterns characterized by emotional disconnection, isolation, and shame; or others characterized by dependency, loss of autonomy and self-confidence. Beliefs and perceptions about the disease activate needs and patterns of cognitive, emotional and relational responses (maladaptive schemas), being both predictive variables of greater or lesser adjustment and global perception of self well-being.

Keywords: Breast cancer, quality of life, early maladaptive schemas,beliefs

Sumario: 1. Introducción 2. Método 3. Análisis de datos 4. Resultado 5. Discusión 6. Conclusión 7. Referencias bibliográficas

Como citar: Protesoni AL, Grille S. Variables predictivas de calidad de vida en mujeres con cáncer de mama. Psicooncología 2021; 18: 261-275, https://dx.doi.org/10.5209/psic.77754

\section{Introducción}

El cáncer de mama es una enfermedad caracterizada por el crecimiento anómalo e incontrolado de células epiteliales de mama, ocasionada por alteraciones genéticas y epigenéticas ${ }^{(1)}$. A nivel mundial está considerada como una de las enfermedades que genera más alerta en las mujeres, por su alta incidencia y mortalidad, convirtiéndose en un tema clave para la salud pública.

En Uruguay, dentro de las patologías oncológicas es la de mayor prevalencia. Según cifras de la Comisión Honoraria de Lucha Contra el Cáncer, una de cada diez mujeres puede desarrollar cáncer de mama a lo largo de su vida ${ }^{(2)}$.

El diagnóstico de cáncer de mama suele aparecer de modo disruptivo, generando una alteración en la homeostasis. Se activan procesos de duelo oncológico y malestar durante la enfermedad y posteriormente ${ }^{(3)}$. El diagnóstico opera como un suceso adverso que se desarrolla desde el interior del cuerpo aunque no se lo reconoce como propio y produce una vivencia de amenaza vital que puede llevar a la paciente a una situación de desorganización. A su vez, la mujer transita una sucesión de eventos que crean un entorno disruptivo ocasionado por los tratamientos invasivos y prolongados en el tiempo y los posteriores controles que operan como una marca ("espada de Damócles") aún en fase de sobrevivencia o remisión ${ }^{(4)}$. Este estudio parte del supuesto que ante la enfermedad las mujeres activan una serie de esquemas desadaptativos tempranos (EDT), vale decir, patrones de pensamiento y emociones, que condensan sensaciones, recuerdos, cogniciones, referidas a sí mismas y a su relación con los demás. Los EDT, así como las representaciones cognitivas y emocionales que elabora la mujer sobre el cáncer de mama, pueden incidir en las estrategias de afrontamiento de la enfermedad, en la toma de decisiones, el ajuste o la adaptación a la enfermedad ${ }^{(5,6)}$. Por consiguiente, tales procesos se considera que 
impactan en la calidad de vida durante el proceso de enfermedad, entendiendo por tal la distancia entre la percepción subjetiva con respecto a su salud y la expectativa trazada ${ }^{(7-9)}$. Varias investigaciones indican que entre el $35 \%$ y el $38 \%$ de los pacientes con cáncer desarrollan malestar emocional significativo durante la enfermedad ${ }^{(10)}$. Algunos estudios muestran que es especialmente importante el malestar emocional (principalmente la ansiedad y la depresión) que se produce en las etapas iniciales de la enfermedad, en la fase de supervivencia aguda (el período entre el diagnóstico y los tratamientos oncológicos primarios) y en la fase de supervivencia extendida (período entre el fin de los tratamientos oncológicos y los primeros seguimientos de mantenimiento) ${ }^{(11)}$. Las primeras fases son las más críticas en el proceso de adaptación a la enfermedad y adherencia psicosocial a los tratamientos ${ }^{(12)}$. El malestar es una dimensión de la calidad de vida, otras dimensiones se vinculan con aspectos físicos, funcionales, sociales, espirituales. Hay numerosos estudios que valoran la CVRS en mujeres con cáncer de mama y su relación con variables sociodemográficas, clínico-biológicas, patrones de funcionamiento, cualidades psíquicas, creencias y percepciones en torno a la enfermedad, así como con las estrategias de afrontamiento. Con respecto a la CVRS en pacientes con patologías mamarias, algunos estudios observan que a nivel global, esta es valorada por las pacientes por encima de la media de las escalas utilizadas ${ }^{(13-16)}$. Pero también hay otros estudios que plantean que estos valores descienden ante la quimioterapia ${ }^{(17)} \mathrm{o}$ ante la mastectomía radical sin reconstrucción ${ }^{(18-20)}$. Por otra parte, a pesar que la valoración de la CVRS pueda ser buena, las mujeres perciben impactos negativos en aspectos tales como la percepción de vulnerabilidad, la preocupación por la salud, su imagen corporal ${ }^{(19,14)}$. Reich y Remor ${ }^{(20)}$ encontraron en mujeres uruguayas diagnosticadas e intervenidas quirúrgicamente por cáncer de mama que aquellas que posteriormente tuvieron reconstrucción mamaria presentaron niveles significativamente menores de estrés percibido y síntomas depresivos, así como una mejor CVRS. Dapueto ${ }^{(21)}$ también en población oncológica uruguaya encontró que la única variable biomédica relevante como determinante de la CVRS global, y especialmente del bienestar físico, fue el estadio tumoral. Con respecto a la modalidad de tratamiento, encontró que la quimioterapia repercutía sobre el bienestar físico y emocional; la radioterapia, sobre la dimensión de bienestar social y familiar. La combinación de tratamientos (quimioradio-hormonoterapia) no parece ocasionar un efecto significativo sobre la CVRS global ni sobre el bienestar funcional. Por lo tanto se puede decir, a partir de los resultados de investigaciones recabadas, que la calidad de vida de las mujeres con cáncer de mama es un constructo relevante para las ciencias de la salud. Dicho constructo está atravesado por diferentes factores entre los que se encuentra el estadio de la enfermedad, así como la edad de la paciente, el nivel socioeducativo y económico, el apoyo sociofamiliar, el estado anímico y la asistencia recibida.

El estudio realizado busca determinar el valor predictivo que tiene sobre la CVRS de las mujeres con cáncer de mama en tratamiento, las variables sociodemográficas, clínicas (tipo de tratamiento) o psicológicas tales como los esquemas desadaptativos tempranos (EDT), las creencias y percepciones sobre la enfermedad y las estrategias de afrontamiento utilizadas. Se parte del supuesto que los esquemas desadaptativos tempranos y las creencias sobre la enfermedad determinan las estrategias de afrontamiento durante el tratamiento del cáncer de mama, impactando en la calidad de vida. Los antecedentes de investigaciones sobre el tema permiten suponer que al trabajar sobre los esquemas desadaptativos tempranos y las creencias en torno 
a la enfermedad una vez que se identifican, es posible incidir en las estrategias de afrontamiento y, por consiguiente, en la CVRS.

\section{Método}

Se realizó un estudio descriptivo, correlacional, de subtipo predictivo ${ }^{(22)}$ con el fin de determinar la asociación y el valor predictivo que tienen sobre la calidad de vida relacionada con la salud (variable de resultado), las características sociodemográficas y clínicas de las mujeres (tipos de tratamientos oncoespecíficos), los esquemas desadaptativos tempranos, las creencias y percepciones sobre la enfermedad y las estrategias de afrontamiento (variables asociadas).

Muestra, procedimiento de recolección de datos y aspectos éticos:

Se seleccionó una muestra de 228 pacientes adultas en tratamiento ambulatorio por cáncer de mama, usuarias del sistema público de asistencia (Hospital de la Mujer, Instituto Nacional del Cáncer, Hospital de Clínicas) ubicado en Montevideo, Uruguay. Se realizaron entrevistas presenciales con investigadores entrenados, en las policlínicas luego de la consulta médica. Se invitó a las pacientes a participar voluntariamente de la investigación, se les informó del estudio y se obtuvo el consentimiento. Se procedió a realizar las entrevistas cara a cara, aplicando los protocolos que llevaron un promedio de 30 minutos.

\section{Instrumentos}

Se utilizaron los siguientes instrumentos para la recolección de datos:

- Formulario de datos socio demográficos y clínicos con el fin de valorar las características de la muestra;

- Cuestionario FACT-B (4a Versión) para pacientes oncológicos, que evalúa la Calidad de Vida Relacionada con la Salud en poblaciones adultas, adaptado al Uruguay. La escala está compuesta por 36 ítems distribuidos en dos componentes: uno general (27 ítems) y otro relacionado con los síntomas del cáncer de mama (nueve ítems). El cuestionario evalúa cinco dominios: bienestar físico ( 7 ítems), bienestar social/familiar ( 7 ítems), bienestar emocional (6 ítems), bienestar funcional (7 ítems) y una subescala específica relacionada con la enfermedad y con el tratamiento del cáncer de mama (9 ítems). Cada ítem se puntúa en una escala tipo Likert con cinco niveles que van de 0 (nada) hasta cuatro (muchísimo). El análisis de confiabilidad muestra una alta consistencia interna como lo indican los coeficientes alfa de Cronbach que van desde 0.-78 a $0 .-91^{(13)}$.

- Test de Esquemas Tempranos (Young Schema Questionnaire YSQ-CEY-VR) de Young y Brown, del cuestionario en su forma corta (YSQ-S3), adaptado al Uruguay, en su tercera edición traducida por Tineo ${ }^{(23)}$. Consta de 75 ítems que evalúan 15 EDT. Cada pregunta es evaluada por el paciente en términos de intensidad en una escala del 1 («totalmente falso») al 6 («me describe perfectamente»). Cada EDT es medido por 5 preguntas, siendo el máximo puntaje 30. Valora los patrones disfuncionales, formados por recuerdos, emociones, pensamientos y sensaciones corporales que hacen referencia al análisis de uno mismo y de las 
relaciones con los demás. Esos patrones se originan durante la infancia a partir de representaciones basadas en la realidad y se van elaborando a lo largo de la vida, siendo disfuncionales posteriormente en grado significativo. El test discrimina los EDT, agrupados en 5 dominios. Según el estudio realizado por Cordero y Tiscornia $^{(24)}$ (2014) en población uruguaya, (aunque no específicamente con neoplasias) tiene una fiabilidad determinada por el coeficiente alfa de Cronbach que oscila entre 0,74 y 0,89 .

- Cuestionario de percepción de enfermedad en su versión abreviada: (The Brief Illness Perception Questionnaire, IPQ-R) desarrollado originalmente por Broadbent, Petrie, Main y Weinman ${ }^{(2)}$, con el fin de valorar las creencias y percepciones en torno a la enfermedad oncológica. Este cuestionario está basado en el Modelo de Autorregulación de sentido común de Leventhal. El cuestionario está formado por tres dimensiones que contienen ocho ítems que se responden utilizando un sistema de respuesta tipo Likert que va de 0-10.

- Escala Brief COPE de Carver, et.al. ${ }^{(26)}$, para evaluar las estrategias de afrontamiento en versión abreviada, ampliamente utilizada en investigaciones en salud. El instrumento consta de 12 subescalas con dos preguntas cada una, conformando un total de 24 ítems con un formato de respuesta tipo Likert que va de 1 "no hice esto en lo absoluto" a 4 "hice esto con mucha frecuencia". La escala permite agrupar las estrategias categorizando aquellas centradas en el problema, en la emoción o evitativas. Se tomó para tal fin la categorización realizada por Morán, et.al. ${ }^{(27)}$ y por Reich y Remor ${ }^{(20)}$. El alfa de Cronbach global de la escala fue fiable de $0.84^{(20)}$.

\section{Análisis de datos}

Luego de valorar cada uno de los instrumentos y codificar los datos sociodemográficos se transfirieron los resultados a una matriz utilizando el paquete estadístico SPSS 21. Se realizaron análisis descriptivos, análisis de estadística inferencial, análisis de regresión lineal múltiple y análisis de regresión jerárquica incorporando todas las variables predictoras en pasos sucesivos, a los efectos de estudiar el peso relativo de cada una de ellas sobre la calidad de vida. Dado que los datos que se utilizaron en esta investigación no están organizados en un mismo nivel, sino que están estructurados de manera jerárquica (variables sociodemográficas y clínicas, esquemas desadaptativos tempranos, creencias y percepción de la enfermedad, estrategias de afrontamiento) se decidió hacer un análisis de regresión lineal múltiple jerárquica. A tales efectos se elaboraron modelos de ecuaciones lineales con fines predictivos. Se tomó como variable dependiente la CVRS a nivel global. Se utilizó como método introducir sucesivamente los bloques, calculándose los coeficientes de determinación $\mathrm{R}^{2}$, el cambio en el $\mathrm{R}^{2}$, los coeficientes estandarizados Beta con sus valores de significancia estadística. Se elaboraron 4 bloques con las variables explicativas en los cuales se colocaron: variables sociodemográficas y clínicas en un bloque, variables psicológicas tales como: EDT en un segundo bloque, estrategias de afrontamiento en un tercer bloque y Creencias y percepción de enfermedad en un cuarto bloque. En todos los casos se consideró como estadísticamente significativo un valor de $\mathrm{p}$ menor 0,05 . 


\section{Resultados}

Características sociodemográficas y clínico-biológicas:

La edad promedio de la muestra se ubica en los 58 años, abarcando desde los 32 a los 89 años. La mayoría de las mujeres se encuentran casadas, han tenido hijos y conviven con familiares. El 37,6\% se encuentra en tratamiento de quimioterapia y el $31,7 \%$ en tratamientos de hormono e inmunoterapia. El 14,1 \% de las mujeres se encontraba en control por cirugía reciente, habiendo sido intervenidas el 95,6\% de la muestra. De ellas, la mayoría tiene conservada la mama (lumpectomía, mastectomía parcial), aunque un porcentaje muy bajo $(7,9 \%)$ se realizó reconstrucción mamaria (Tabla 1$)$.

Tabla 1. Tipo de Cirugía realizada para tratamiento del cáncer de mama

\begin{tabular}{ll}
\hline & $\mathrm{N}(\%)$ \\
\hline Cirugía con menor afectación de imagen & $\mathbf{1 3 1}(\mathbf{6 8 , 6} \%)$ \\
Lumpectomía & $47(24,6 \%)$ \\
Mastectomía parcial & $69(36,1 \%)$ \\
Mastectomía radical con reconstrucción & $15(7,9 \%)$ \\
Mastectomía radical sin reconstrucción & $\mathbf{6 0}(\mathbf{3 1 , 4 \% )}$ \\
\hline
\end{tabular}

Calidad de vida relacionada con la salud y variables clínico-biológicas

En la escala global de calidad de vida (que va de 0 a 148) se registró una mediana de FACT-B Global de 105 (RIQ 82-118). Los diferentes tipos de tratamientos onco específicos no registraron diferencias significativas en la CVRS, sin embargo los tipos de cirugía por las que pasaron las mujeres (conservadora o no) si registraron diferencias significativas en la CVRS. La valoración que hacen las mujeres con mastectomía sin reconstrucción se encontró sensiblemente descendida con respecto a los otros tipos de cirugía, de ahí que se eligió la variable cirugía para evaluar su predicción en la CVRS (Tabla 2, Figura 1).

Tabla 2. FACT-B en función del tipo de cirugía realizada

\begin{tabular}{llllll}
\hline $\mathrm{n}=203$ & $\begin{array}{c}\text { Lumpectomía } \\
\text { Mediana (RIQ)* }\end{array}$ & $\begin{array}{c}\text { Mastectomía } \\
\text { parcial } \\
\text { Mediana (RIQ)* }\end{array}$ & $\begin{array}{c}\text { Mastectomía radical } \\
\text { con reconstrucción } \\
\text { Mediana (RIQ)* }\end{array}$ & $\begin{array}{c}\text { Mastectomía radical } \\
\text { sin reconstrucción } \\
\text { Mediana (RIQ)* }\end{array}$ & $\begin{array}{c}\text { Valor } \\
\text { de } \mathrm{p}^{* *}\end{array}$ \\
\hline $\begin{array}{l}\text { Estado } \\
\text { Físico }\end{array}$ & $25(20-27)$ & $20,0(15-25)$ & $24(16-26)$ & $21,5(14,5-25)$ & 0,009 \\
\hline $\begin{array}{l}\text { Ambiente } \\
\text { familiar y social }\end{array}$ & $20(15-24)$ & $20,5(15-26)$ & $20(12-25)$ & $19,(14,5-22)$ & 0,45 \\
\hline $\begin{array}{l}\text { Estado } \\
\text { emocional }\end{array}$ & $18(14-21)$ & $18(14-22)$ & $18(15-23)$ & $17,5(12-23)$ & 0,81 \\
\hline
\end{tabular}




\begin{tabular}{llllll}
\hline $\begin{array}{l}\text { Funcionamiento } \\
\text { personal }\end{array}$ & $21(16-22)$ & $20(13-25)$ & $20(14-25)$ & $21(16,5-24)$ & 0,98 \\
\hline $\begin{array}{l}\text { Síntomas } \\
\text { vinculados } \\
\text { cáncer de mama }\end{array}$ & $25(20-31)$ & $26,0(22,0-29,0)$ & $27(22-34)$ & $24,0(22,0-31)$ & 0,88 \\
\hline $\begin{array}{l}\text { FACT- } \\
\text { Global }\end{array}$ & $107(85-119)$ & $104,5(79-118)$ & $115(81-12)$ & $103(86-113)$ & 0,63 \\
\hline
\end{tabular}

*RIQ: rango intercuartil

**Prueba Kruskal-Wallis

Figura 1. Se muestra la subescala Estado físico del FACT-B, en función de tipo de cirugía. Se expresa en mediana \pm error standard $(p=0,009)$.

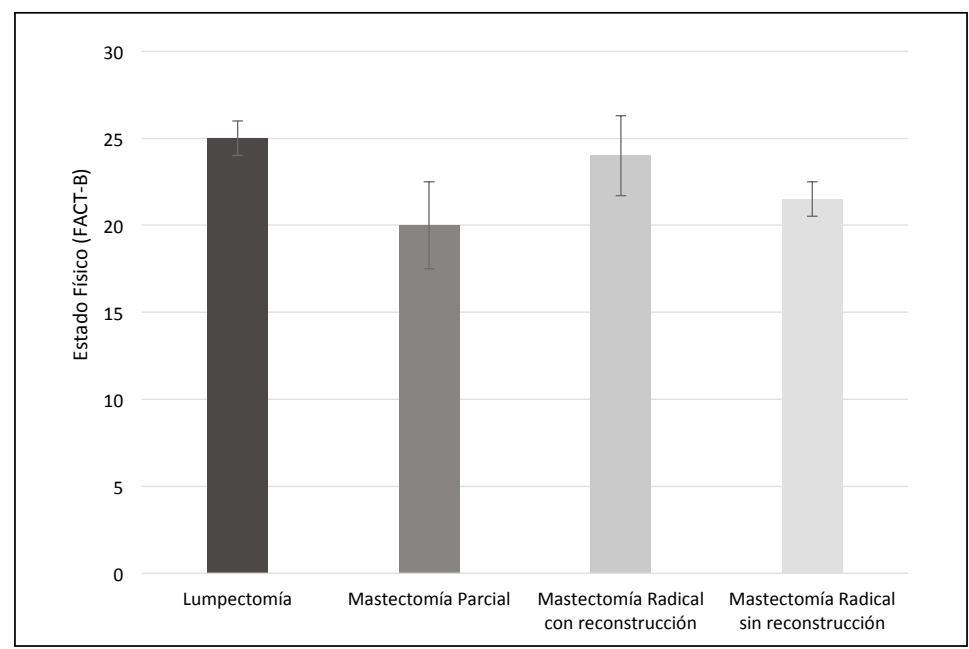

Esquemas desadaptativos tempranos y calidad de vida

Los EDT que aparecen más activos y por encima de la media para su rango son el Autosacrificio 24 (17-29) y el de Metas Inalcanzables 18 (13-23,7). El EDT de Autosacrificio se caracteriza por la dedicación excesiva sobre la satisfacción de las necesidades de los demás en situaciones cotidianas, en detrimento de la propia satisfacción. La persona asume comportamientos que tienen como fin evitar causar dolor a los demás. El EDT de Metas inalcanzables refiere a creencias relacionadas con un elevado esfuerzo que la persona debe hacer para alcanzar estándares muy altos de conducta y desempeño. Esta creencia provoca sentimientos de presión y una actitud hipercrítica hacia sí mismo y hacia los demás.

Se realizó análisis de correlación lineal (Spearman) entre la variable de CVRS FACT- B global y las diferentes variables de EDT, con el fin de evaluar la relación entre CVRS y EDT. Se obtuvo una correlación moderada e inversa entre la CVRS a nivel Global y los esquemas de Deprivación Emocional y Aislamiento Social. Se podría plantear que la situación de enfermedad y los tratamientos parecen activar necesidades insatisfechas de seguridad, estabilidad, cuidado y aceptación. Estas 
necesidades llevan a poner en acción esquemas en los que aparecen dificultades en las relaciones generadas por sentimientos de inseguridad, desconfianza en los vínculos y temores al abandono.

Posteriormente, se realizó un análisis de regresión lineal múltiple utilizando todas las variables de EDT que eran estadísticamente significativas en la correlación lineal (todas excepto sobrevaloración). Se utilizó el método introducir. El análisis excluyo las variables Dominio 1 y Dominio 2.

En la tabla 3 se muestran los resultados de la regresión lineal múltiple. Se observa que las variables que mostraron relación estadística en explicar FACT-Global fueron Deprivación emocional, Abandono, Imperfección/vergüenza, Vulnerabilidad, Apego, Dominio 3 y Dominio 4. Todas mostraron una correlación de tipo inversa con el FACT-Global, excepto Dominio 3 y Dominio 4 con una correlación positiva con FACT-Global. El modelo global fue estadísticamente significativo.

Tabla 3. Análisis de regresión lineal múltiple entre FACT-Global y los diferentes esquemas desadaptativos tempranos

\begin{tabular}{lcccc}
\hline & $\beta$ & $\begin{array}{c}\text { IC 95\% límite } \\
\text { inferior }\end{array}$ & $\begin{array}{c}\text { IC 95\% límite } \\
\text { superior }\end{array}$ & Valor de p \\
\hline Deprivación emocional & $-0,172$ & $-0,965$ & $-0,114$ & 0,0001 \\
Abandono & $-0,053$ & $-0,551$ & 0,229 & 0,013 \\
Desconfianza abuso & $-0,125$ & $-0,913$ & 0,043 & 0,417 \\
Aislamiento social & $-0,195$ & $-1,420$ & $-0,177$ & 0,074 \\
Imperfección/vergüenza & $-0,006$ & $-0,793$ & 0,724 & 0,012 \\
Fracaso & 0,026 & $-0,436$ & 0,679 & 0,928 \\
Dependencia & $-0,206$ & $-1,973$ & $-0,487$ & 0,668 \\
Vulnerabilidad & $-0,198$ & $-1,137$ & $-0,265$ & 0,001 \\
Apego & $-0,055$ & $-0,775$ & 0,250 & 0,002 \\
Sometimiento & $-1,717$ & $-27,556$ & 10,721 & 0,314 \\
Autosacrificio & $-2,399$ & $-27,568$ & 10,465 & 0,387 \\
Metas inalcanzables & $-0,023$ & $-0,539$ & 0,370 & 0,376 \\
Insuficiente autocontrol & $-0,408$ & $-2,452$ & $-0,804$ & 0,714 \\
Dominio 3 & 0,369 & 0,317 & 1,243 & 0,000 \\
Dominio 4 & 3,381 & $-10,413$ & 27,630 & 0,001 \\
Dominio 5 & $-0,087$ & $-0,694$ & 0,111 & 0,373 \\
\hline
\end{tabular}

Modelo global: $\mathrm{F}=15,120, \mathrm{p}=0,0001 ; \mathrm{R}^{2}=0,550 ; \mathrm{R}^{2}$ corregida $=0,510$

Creencias y percepciones en relación a la enfermedad y calidad de vida

Con respecto a la percepción global sobre la enfermedad, se registró en las mujeres una percepción de amenaza de la enfermedad con una mediana de 27,8 $\pm 16,7$, ubicándose por debajo de la media en la escala. Si se considera la desviación se 
observa que las respuestas tienen una gran dispersión que hace que algunas mujeres tengan una percepción muy amenazante, mientras que otras minimicen sus efectos. En el análisis de correlación lineal simple todos los valores del IPQ-R guardaron relación significativa con la CVRS a nivel global. (Tabla 4, figura 2)

Tabla 4. Análisis de correlación de IPQ-R con FACT-global

\begin{tabular}{lcc}
\hline $\begin{array}{l}\text { Percepción y creencias sobre la } \\
\text { enfermedad }\end{array}$ & r Spearman & Valor de p \\
\hline Consecuencias & $-0,55$ & 0,0001 \\
Duración de la enfermedad & $-0,34$ & 0,0001 \\
Control sobre la enfermedad & 0,15 & 0,027 \\
Control sobre el tratamiento & 0,33 & 0,0001 \\
Identidad & $-0,49$ & 0,0001 \\
Conciencia la enfermedad & $-0,39$ & 0,0001 \\
(preocupación) & 0,22 & 0,0013 \\
Coherencia sobre la enfermedad & $-0,48$ & 0,0001 \\
Respuesta emocional & $-0,60$ & 0,0001 \\
IPQ-R Global & & \\
\hline Representaciones cognitivas $\boldsymbol{y}$ & $-0,44$ & 0,0001 \\
emocionales & $-0,48$ & 0,0001 \\
\hline Representación Cognitiva & & \\
Representación Emocional & & \\
\hline
\end{tabular}

Figura 2. Correlación lineal entre Fact-Global y IPQ-R Global.

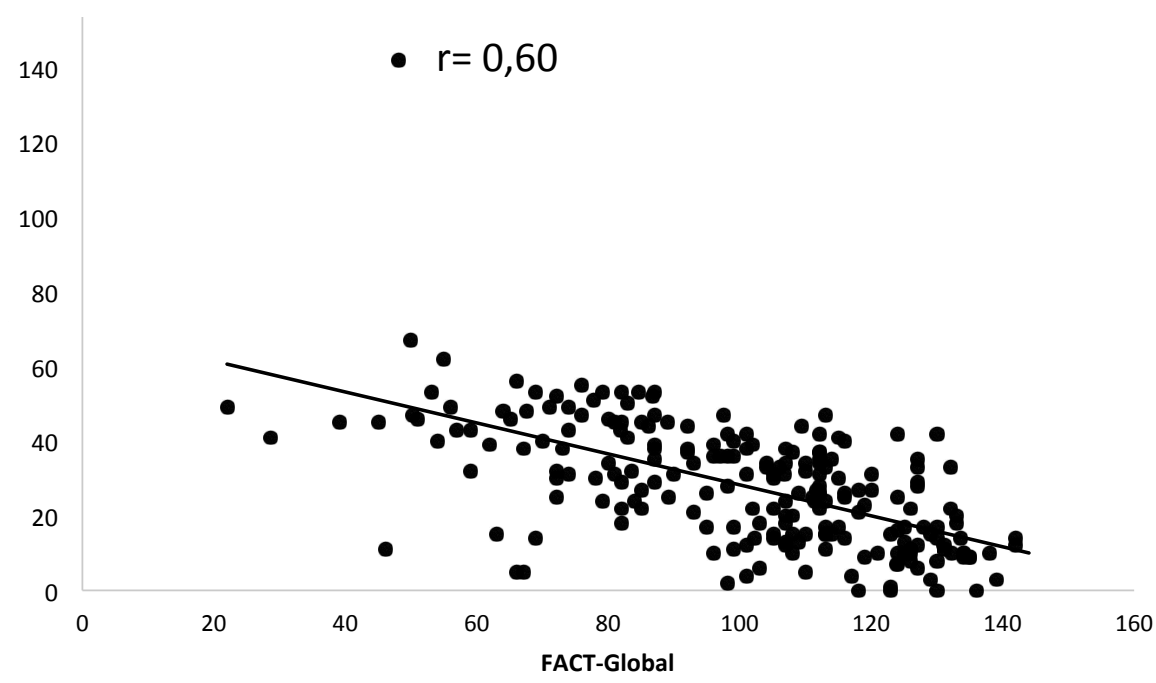


Posteriormente, se realizó un análisis de regresión lineal múltiple, utilizando todas las variables del IPQ-R, ya que resultaron estadísticamente significativas en la correlación lineal. Se utilizó el método introducir. El análisis excluyó la variable Representación emocional en tanto se registró que no agrega datos para predecir la CVRS. En la tabla V se muestran los resultados de la regresión lineal múltiple. El modelo global fue estadísticamente significativo.

Tabla 5. Análisis de regresión lineal múltiple entre FACT-Global y las diferentes variables de IPQ-R

\begin{tabular}{lcccc}
\cline { 2 - 5 } & $\beta$ & $\begin{array}{c}\text { IC 95\% límite } \\
\text { inferior }\end{array}$ & $\begin{array}{c}\text { IC 95\% límite } \\
\text { superior }\end{array}$ & $\begin{array}{c}\text { Valor } \\
\text { de } \mathrm{p}\end{array}$ \\
\hline $\begin{array}{l}\text { Percepción y creencias sobre la } \\
\text { enfermedad }\end{array}$ & & $-5,9$ & \\
\hline Consecuencias & $-0,57$ & $-4,5$ & $-0,84$ & 0,0001 \\
Duración de la enfermedad & $-0,37$ & $-3,7$ & $-0,47$ & 0,012 \\
Control sobre la enfermedad & $-0,26$ & $-5,6$ & $-2,04$ & 0,0001 \\
Identidad & $-0,52$ & $-0,176$ & 3,11 & 0,08 \\
Conciencia la enfermedad & $-0,22$ & $-1,5$ & 0,90 & 0,61 \\
(preocupación) & $-0,035$ & $-0,69$ & 0,36 & 0,53 \\
Coherencia sobre la enfermedad & $-0,11$ & & & \\
IPQ Global & & 0,66 & 3,4 & 0,004 \\
\hline $\begin{array}{l}\text { Representaciones cognitivas } \boldsymbol{y} \\
\text { emocionales }\end{array}$ & 0,56 & $-2,04$ & 0,11 & 0,080 \\
\hline $\begin{array}{l}\text { Representación Cognitiva } \\
\text { Representación Emocional }\end{array}$ & $-0,27$ & & & \\
\hline
\end{tabular}

Modelo global: $\mathrm{F}=20,05, \mathrm{p}=0,0001 ; \mathrm{R}^{2}=0,47 ; \mathrm{R}^{2}$ corregida $=0,44$

Estrategias de afrontamiento y calidad de vida

Con respecto a las estrategias de afrontamiento, se registró un uso de gran variedad de estrategias con similar frecuencia. Esto denota que las pacientes no utilizan una única estrategia para hacer frente a la situación estresante. No se encontraron correlaciones significativas entre las estrategias de afrontamiento y la calidad de vida, ni a nivel global ni en cada una de las sub escalas.

Variables sociodemográficas, clínico-biológicas, psicológicas y calidad de vida:

Para determinar la relación que existe entre la variable dependiente CVRS y un conjunto de variables independientes se realizó un análisis de regresión lineal múltiple jerárquica. Se tomó como variable dependiente la CVRS a nivel global y se elaboraron 4 bloques con las variables explicativas. 
- Dentro del primer bloque se tomaron las variables sociodemográficas tales como: la edad, el lugar de residencia (interior $\square$ capital), el nivel educativo, la religión y el núcleo familiar. Dentro de las variables clínicas se consideró el tipo de cirugía recibida (radical o conservadora), en tanto se encontró que la valoración que hacen las mujeres con mastectomía sin reconstrucción se encontró sensiblemente descendida con respecto a los otros tipos de cirugía. En los bloques siguientes se colocaron las variables psicológicas:

- En el segundo bloque: se consideraron los 5 dominios en los que se agrupan los 15 EDT estudiados (1. Desconexión y rechazo; 2. Autonomía y desempeño deteriorados; 3. Límites inadecuados; 4. Tendencia hacia los otros; 5. Hipervigilancia e inhibición).

- En el tercer bloque: se agruparon los estilos de afrontamiento de acuerdo a la caracterización en tipos de afrontamiento centrados en la emoción, centrados en el problema y evitativos.

- En el cuarto bloque se colocó el índice global de percepción de gravedad sobre la enfermedad.

El análisis de regresión lineal múltiple jerárquica permitió examinar la relación predictiva entre las variables sociodemográficas, clínicas y psicológicas, con la calidad de vida percibida.

Se calculó el coeficiente de determinación del $\mathrm{R}^{2}$, la varianza en el $\mathrm{R}^{2}$, los coeficientes estandarizados $\mathrm{B}$, y el valor de $\mathrm{p}$ (Tabla 6).

Tabla 6. Regresión lineal múltiple jerárquica con FACT-Global como variable dependiente

\begin{tabular}{lccc}
\hline Modelo & R cuadrado & $\begin{array}{c}\text { Cambio en R } \\
\text { cuadrado }\end{array}$ & $\mathrm{p}$ \\
\hline 1- sociodemográficas / clínicas & 0,017 & 0,017 & 0,811 \\
2- Dominios de EDT & 0,490 & 0,473 & 0,0001 \\
3- Estilos de afrontamiento & 0,507 & 0,017 & 0,130 \\
4-Creencia y percepción de enfermedad & 0,606 & 0,100 & 0,0001 \\
\hline
\end{tabular}

Se realizaron diversas regresiones jerárquicas alternando el orden de los bloques. (Tabla 7)

Tabla 7. Regresión lineal múltiple jerárquica con FACT-Global como variable dependiente

\begin{tabular}{clcc}
\hline Modelo & Variables & $\boldsymbol{\beta}$ & $\mathbf{p}$ \\
\hline \multirow{4}{*}{1} & Edad & 0,033 & 0,685 \\
& Primaria completa & 0,056 & 0,477 \\
& Departamento & 0,091 & 0,230 \\
& Religión & $-0,066$ & 0,383 \\
& Cirugía radical & $-0,030$ & 0,686 \\
& Vive sola & 0,030 & 0,702 \\
\hline
\end{tabular}




\begin{tabular}{clcc}
\hline Modelo & Variables & $\boldsymbol{\beta}$ & $\mathbf{p}$ \\
\hline \multirow{2}{*}{$\mathbf{2}$} & Dominio 1 & $-0,453$ & 0,000 \\
& Dominio 2 & $-0,328$ & 0,000 \\
& Dominio 3 & 0,063 & 0,377 \\
& Dominio 4 & 0,030 & 0,682 \\
& Dominio 5 & $-0,090$ & 0,210 \\
\hline \multirow{2}{*}{3} & Afrontamiento & 0,149 & 0,021 \\
& centrado Emoción & & \\
& Afrontamiento & $-0,054$ & 0,392 \\
& centrado Problema & $-0,043$ & 0,479 \\
\hline $\mathbf{4}$ & Afrontamiento Evitativo & $-0,382$ & 0,000 \\
\hline
\end{tabular}

Se colocó primero el bloque de creencias y percepción global de la enfermedad (IPQ-R) sobre los dominios de los EDT y se observó que ambos tienen valor predictivo sobre CVRS, lo que hace pensar que las creencias y percepciones de la enfermedad influyen sobre los EDT, midiendo variables similares.

Los resultados del análisis de predicción ponen de manifiesto que tanto las creencias y percepción sobre la enfermedad como los dominios 1 y 2 de los EDT son buenos predictores de la CVRS, explicando el $60 \%$ de la varianza en la CVRS. Las estrategias de afrontamiento y las variables socio demográficas o clínicas seleccionadas no producen alteraciones significativas en la CVRS.

\section{Discusión}

En función de los resultados obtenidos se puede concluir que las variables sociodemográficas tales como la edad, el mayor o menor nivel educativo, el residir en la capital o el interior, el ser o no creyente, no tienen un valor predictivo sobre la calidad de vida durante la enfermedad.

Con respecto a las variables clínico-biológicas, la cirugía no conservadora ha sido en este estudio la que se ha valorado con mayor impacto en la CVRS (especialmente en la escala física). Aun así, esta no llega a constituirse en un factor predictor de la valoración de la calidad de vida global de la mujer.

Sin embargo, la percepción de bienestar en la calidad de vida durante la enfermedad parecería asociarse con el papel que juegan ciertas variables psicológicas. El estudio de regresión lineal múltiple jerárquica reafirma los resultados obtenidos de correlaciones moderadas e inversas con los esquemas del dominio 1: Desconexión y rechazo (abandono, desconfianza-abuso, deprivación emocional, imperfecciónvergüenza y aislamiento social) y 2: Autonomía y desempeño deteriorado (dependencia-incompetencia, vulnerabilidad al daño o enfermedad, apego-identidad no desarrollada y fracaso).

La percepción de bienestar durante la enfermedad y los tratamientos se deteriora sensiblemente cuanto más se activan necesidades insatisfechas de seguridad, estabilidad, cuidado y aceptación. Estas necesidades ponen en acción esquemas en los que prevalecen sentimientos de inseguridad, desconfianza y temores al abandono. En menor medida, pero con un valor significativo también, se encontró que la percepción de bienestar 
se deteriora cuanto más se activan temores a la pérdida en autonomía, competencia e independencia. La calidad de vida se asocia con la percepción de vulnerabilidad durante la enfermedad, de dependencia y fracaso. Al activarse tales esquemas aumentan los requerimientos de apoyo emocional y los temores al daño, dificultando el establecimiento de vínculos de apego seguros y confiables durante el tratamiento.

Por otra parte, también se encontró que las creencias y percepciones sobre la enfermedad tienen valor predictivo en la valoración del bienestar percibido. Cuanto más amenazante y grave es percibida la enfermedad, más se ve afectada la valoración de la CVRS registrándose un deterioro en ella. Éstos resultados son coincidentes con los obtenidos por Giraldo-Mora y Arangano ${ }^{(28)}$, Rozema et. al ${ }^{(29)}$ y Gercovich, et. al (30) quienes también reportan una asociación entre percepción y creencias sobre la enfermedad y la CVRS.

\section{Conclusiones}

En este estudio se encontró que la percepción de bienestar en la calidad de vida durante la enfermedad, parecería asociarse con el papel que juegan ciertas variables psicológicas y el tipo de cirugía. En ese sentido, se encontró que las creencias y percepciones sobre la enfermedad tienen valor predictivo en la valoración del bienestar percibido. Cuanto más amenazante y grave es percibida la enfermedad, cuanto más síntomas físicos, emocionales y consecuencias sociales compromete la enfermedad, las mujeres valoran más deteriorada su CVRS. Las creencias y percepciones que las mujeres elaboran sobre la enfermedad activan necesidades y pautas de respuestas cognitivas, emocionales, relacionales (esquemas desadaptativos) que permiten mayor o menor ajuste y percepción global de bienestar. Se entiende que las creencias y percepciones activan los esquemas desadaptativos tempranos y ambos son predictivos de la CVRS.

Las mujeres con una percepción amenazante de la enfermedad tienden a activar pautas de funcionamiento caracterizadas por la desconexión emocional, el aislamiento, la vergüenza; u otras caracterizadas por la dependencia, la pérdida de autonomía y confianza en sí mismas. Estos patrones de funcionamiento adquieren valor predictivo sobre la calidad de vida, incidiendo en la percepción de deterioro de esta.

Estos resultados permiten pensar que al actuar sobre las creencias y percepciones que tienen las mujeres sobre la enfermedad, así como al actuar sobre los esquemas desadaptativos que se ponen en acción, se podría incidir en la calidad de vida. Estas serían las variables a tener en cuenta para las intervenciones psicooncológicas, por encima de las estrategias de afrontamiento, los aspectos sociodemográficos o clínicos de la enfermedad. Por lo tanto, el estudio permite avalar el supuesto que las creencias sobre la enfermedad, junto con los esquemas desadaptativos tempranos, impactan en la calidad de vida.

\section{Referencias bibliográficas}

1. Peuker A, Armiliato M, Souza L, Kern, E. Causal attribution among women with breast cancer. Psicologia: Reflexão e Crítica 2016; 29:1-6. Disponible en: https://doi. org/10.5209/rev_PSIC.2015.v12.n2-3.51007 
2. Comisión Honoraria de Lucha contra el Cáncer (2019). Situación epidemiológica del Uruguay en relación al cáncer. Incidencia del cáncer en el quinquenio 2011-2015. Tendencia de la mortalidad por cáncer hasta 2017. Montevideo, Registro nacional de cáncer Uruguay. [Acceso 1 de abril de 2021]. Disponible en: http//Informe_RNC_ mayo_2019_Inc_2011-2015_Mort_hasta_2017_-_WEB\%20(1).pdf

3. Benyakar M, Lezica A. Lo traumático: Vol. 2. Clínica y paradoja: abordaje clínico. $1^{\mathrm{a}}$ ed. Buenos Aires: Biblos, 2006.

4. Benyakar, M. Lo disruptivo. $1^{\mathrm{a}}$ ed. Buenos Aires: Biblos, 2003.

5. Parsonnet L. Using the Schema Therapy Model to help patients cope with a cancer diagnosis. 2017. [Acceso 1 de abril de 2021]. Disponible en http://www.schematherapysociety.org/ Schema-Therapy-Model-to-Help-Patients-with-Cancer-Diagnosis/

6. Thomson A, Heyworth J, Girschik G, Slevin T, Saunders C, Fritschi L. Creencias y percepciones sobre las causas del cáncer de mama: un estudio de casos y controles. BMC Res Notes 2014; 7, 558.

7. Bayes R. Psicología oncológica. Barcelona: Martínez Roca; 1991.

8. Crespo M, Cruzado J. La evaluación del afrontamiento: adaptación española del cuestionario COPE con una muestra de estudiantes universitarios. Anál Mod Cond 1997 23: 797-830. Disponible en: https://dialnet.unirioja.es/servlet/articulo?codigo=7071279

9. Stanton A, Danoff-burg S, Huggins M. The first year after breast cancer diagnosis: hope and coping strategies as predictors of adjustment. Psychooncology 2002; 11: 93-102. https://doi.org/10.1002/pon.574

10. Carlson L, Waller A, Mitchell A. Screening for distress and unmet needs in patients with cancer: review and recommendations. J Clin Oncol 2012; 30: 1160-77. https://doi. org/10.1200/JCO.2011.39.5509

11. Faller H, Schuler M, Richard M, Heckl U, Weis J, Küffner R. Effects of psychooncologic interventions on emotional distress and quality of life in adult patients with cancer: systematic review and meta-analysis. J Clin Oncol 2013; 31: 782-93. https://doi. org/10.1200/JCO.2011.40.8922

12. Lam WW, Shing YT, Bonanno GA, Mancini AD, Fielding R. Distress trajectories at the first year diagnosis of breast cancer in relation to 6 years survivorship. Psychooncology 2012; 21:90-9. https://doi.org/ 10.1002/pon.1876

13. Dapueto, J. Campo disciplinar y campo profesional de la psicología médica. Enfoque biopsicosocial de la medicina: 30 años después. Rev. Médica del Uruguay 2016; 32:197-204.

14. Lopes JV, Bergerot CD, Barbosa LR, Calux NM, Elias S, Ashing, KT, Domenico, E. BLD. Impact of breast cancer and quality of life of women survivors. Rev Brasileira de Enfermagem 2018; 71:2916-21. https://doi.org/10.1590/0034-7167-2018-0081

15. Pineda Higuita S., Andrade Mosquera, Montoya Jaramillo, Y. Factores asociados a la calidad de vida en mujeres con cáncer de mama. Medellín 2013. Rev Gerencia y Políticas de Salud 2017; 16:85-95. [Acceso 1 de abril de 2021]. Disponible en: https://www. redalyc.org/pdf/545/54552517006.pdf

16. Recalde M. Samudio M. Calidad de vida en pacientes con cáncer de mama en tratamiento oncológico ambulatorio en el Instituto de Previsión Social en el año 2010. Memorias del Instituto de Investigaciones en Ciencias de la Salud 2012; 10:13-29. Acceso 1 de abril de 2021]. Disponible en: https://revistascientificas.una.py/index.php/RIIC/article/view/121

17. De Castro Figueiredo Pereira Coelho R, Nunes Garcia S, Marcondes L, Jacinto da Silva FA, de Paula A, Puchalski Kalinke L. Impact on the quality of life of women with breast cancer undergoing chemotherapy in public and private care. Investigación y educación en Enfermería 2018; 36(1). https://doi.org/10.17533/udea.iee.v36n1e04 
18. Bajuk L, Reich, M. Calidad de vida relacionada con la salud en mujeres uruguayas con cáncer de mama. Ciencias Psicológicas 2011; 5: 19-30. Disponible en: https://revistas. ucu.edu.uy/index.php/cienciaspsicologicas/article/view/96/85

19. Gargantini A, Casari JM. Imagen corporal y su influencia en la calidad de vida en mujeres con mastectomía. Comparación entre mujeres con reconstrucción inmediata, reconstrucción diferida o sin reconstrucción. Psicooncología 2019; 16:43-60. https://doi. org/10.5209/PSIC.63647

20. Reich M, Remor E. Calidad de vida relacionada con la salud y variables psicosociales: Caracterización de una muestra de mujeres uruguayas con cáncer de mama. Psicooncología 2011; 8:453-71. https://doi.org/10.5209/rev_PSIC.2011.v8.n2-3.37892

21. Dapueto J. Calidad de vida. Marco conceptual, operacionalización y aplicaciones clínicas. Montevideo: Ediciones Universitarias. 2013.

22. Dankhe GL. Investigación y comunicación. En Fernández-Collado C, Dankhe GL, editores. La comunicación humana: ciencia social. México: Mc Graw Hill; 1986. p.385454.

23. Young J, Brown G. Cuestionario de Esquemas Desadaptativos (versión breve, Trad. J. Cid y R. Torrubia, Adaptación para Uruguay L. Tineo). 2008. [Test psicológico. Publicación original de 1995].

24. Cordero S, Tiscornia M. Esquemas desadaptativos tempranos y trastornos de la personalidad: resultados de un estudio piloto. 2014 Tesina. [Acceso 1 de abril de 2021]. Disponible en: http://www.mairatiscornia.com/wp-content/uploads/2013/07/EDTs-y-TPEstudio-piloto-Cordero-Tiscornia.pdf

25. Broadbent E, Petrie K, Main, J, Weinman J. The Brief Illness Perception Questionnaire, IPQ-R.JPsychosomRes2006; 60:631-7.https://doi.org/10.1016/j.jpsychores.2005.10.020

26. Carver C, Scheier M, Weintraub J. Assessing coping strategies: a theoretically based approach. J Pers Soc Psychol 1989; 56:267-83.

27. Morán MC, Landero R, González MT. COPE-28: un análisis psicométrico de la versión en inglés del Brief COPE. Universitas Psychologica 2010; 9:543-52. Disponible en: http:// pepsic.bvsalud.org/scielo.php?script=sci_arttext\&pid=S1657-92672009000200020

28. Giraldo-Mora CV, Arango ME. Representaciones sociales frente al autocuidado en la prevención del cáncer de mama. Investigación y Educación en Enfermería 2009; 27:191200. [Acceso 1 de abril de 2021]. Disponible en: http://www.scielo.org.co/pdf/iee/v27n2/ v27n2a04.pdf

29. Rozema H, Vollnik T, Lechner, L. The role of illness representations in coping and health of patients treated for breast cancer. Psychooncology 2009; 18:849-57. https://doi. org/10.1002/pon.1488

30. Gercovich D, López P, Bortolato D, Margiolakis, Morgenfel M, Rosell L, Gil Deza E. El rol del distrés psicológico en la relación entre percepción de enfermedad y calidad de vida en pacientes con cáncer de mama. Psicooncología 2012; 9:403-14. https://doi. org/10.5209/rev_PSIC.2013.v9.n2-3.40911 